\title{
Measurement and calculating of supersonic ejectors
}

\author{
Lukas Vojta ${ }^{1, *}$, Vaclav Dvorak ${ }^{1}$ \\ ${ }^{1}$ Department of Power Engineering Equipment, Faculty of Mechanical Engineering, Technical University of Liberec, \\ Studentska 2, 46117, Liberec, the Czech Republic
}

\begin{abstract}
This paper deals with numerical and experimental investigation of the flow in an air to air supersonic ejector with constant area mixing chamber. The mixing chamber of previous ejector was completely repaired since a scratch from previous turning had been found. As a result, a new geometry of the mixing chamber was created. Several measurements were conducted with different nozzle position (NP): $1 \mathrm{~mm}, 2 \mathrm{~mm}$ and $3 \mathrm{~mm}$. Furthermore, for a given NP, two different values of stagnation pressure of $200 \mathrm{kPa}$ and $300 \mathrm{kPa}$ at the primary air inlet were investigated in more detail. All numerical simulations were performed in the ANSYS Fluent software. It was found that the influence of the position of the nozzle influences the ejection factor only to a certain extent. For the other parameters of the ejector is also a need to find the optimum position of the nozzle. Repair of the mixing chamber has contributed to reduce the pressure difference at the wall of the mixing chamber.
\end{abstract}

\section{Introduction}

The ejector, as a device working with a high pressure medium, is very constructive simple. The base structure of an ejector has only four parts. The first main part is a convergent-divergent nozzle that allows primary media to reach supersonic speeds. The second part is the suction pipe for suction secondary media, the third is the mixing chamber and the last one is the diffuser.

The convergent-divergence nozzle allows the primary or driving flow to be accelerated up to supersonic speeds. Supersonic velocities are achieved only by changing the geometry of the individual parts of the nozzle. The primary nozzle usually consists of two opposite cones along the direction of the stream. The channel of the nozzle is first tapped (the stream is accelerating to high subsonic velocities, $M \rightarrow 1$ ), the stream reach sonic speed and the flow is chocked in the throat of the nozzle $(\mathrm{M}=1)$ and flow in the divergent part further expands (the stream is further accelerated to supersonic speeds, $M>1)$.

\section{Application of ejectors}

The ejectors are used in areas where there is enough high pressure drive media or the ratio of production costs and operating costs is lower than one. Another advantage is that the ejector is a device without moving parts. Application examples include:

-Developing high vacuum in compressed-air operations.

- Ejector cooling circuits (ECC), where the ejectors replace the compressors. ECC are used where a heat source is available at a higher temperature level. Use of air conditioners in combination with thermal solar panels can be such application.

- Vacuum formation. By setting several ejectors in series, a very low absolute pressure can be created.

-Transport of substances that could damage the moving parts of pumps or fans.

-Pumping liquids from depths that do not allow use of conventional pumps.

- Drive of wind tunnels, especially supersonic.

\section{Theoretical background}

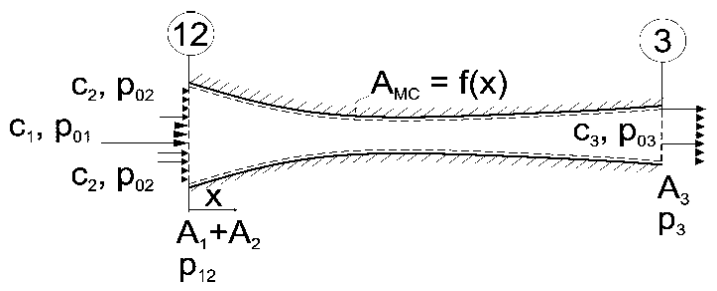

Fig. 1. Control volume of a mixing chamber

The control volume for which we compile the set of balance equations is in Figure 1. We consider the general shape of the mixing chamber. Let us compile the balance equations for the stationary mixing of the ideal fluid [1]. For the general shape of the mixing chamber, three basic conservation equations can be written. The first is the continuity equation and its shape is:

$$
\rho_{1} c_{1} A_{1}+\rho_{2} c_{2} A_{2}=\rho_{3} c_{3} A_{3}
$$

\footnotetext{
*Corresponding author: lukas.vojta@tul.cz
} 
The second of these basic equations of conservationis the momentum equation. We will write it assuming that the pressures of both fluidswill beof the same value $p_{1}=p_{2}=p_{12}$ at the inlet to the mixing chamber.Pressure $p_{12}$ is then called theexpansion pressure. The equation will then be in this form:

$$
\begin{aligned}
& \dot{m}_{1} c_{1}+\dot{m}_{2} c_{2}+p_{12}\left(A_{1}+A_{2}\right)=\dot{m}_{3} c_{3}+p_{3} A_{3}+ \\
& \int_{A_{S K x}} p_{(x)} d A_{S K x} .
\end{aligned}
$$

The third equation is the conservation of energy equation assuming adiabatic flow

$$
\dot{m}_{1} h_{01}+\dot{m}_{2} h_{02}=\left(\dot{m}_{1}+\dot{m}_{2}\right) h_{03}
$$

The Laval nozzle that is used in the experiment is designed for output Mach number $\mathrm{M}=2.07$, which corresponds to the ratio of the critical cross section (throat) and the nozzle outlet cross section $\frac{A}{A_{T}}=$ 1,7902 .

\section{Experimental investigation}

Before start of the measurement, all parts of the ejector were checked. It was found that the nozzle and the mixing chamber are not in the axis. Furthermore, it has been found that there is a scratch in the mixing chamber, which can affect static pressure distribution on the wall of the mixing chamber [5]. The misalignment of the nozzle was solved by simply replacing the screws and the mixing chamber was drilled to a larger dimension. The original diameter of the mixing chamber was $8 \mathrm{~mm}$ and after repair grew the diameter to $8.34 \mathrm{~mm}$. The wall profile, i.e. roughness wall distribution before (Figure 2) and after the repair (Figure 3) are shown below.

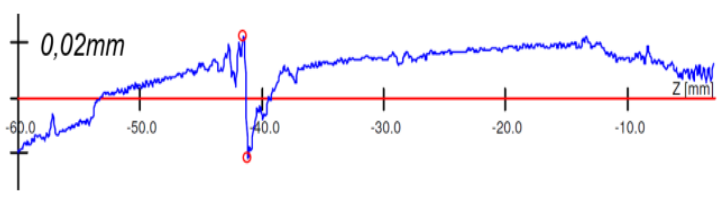

Fig. 2. Wall profile before the repair [4]

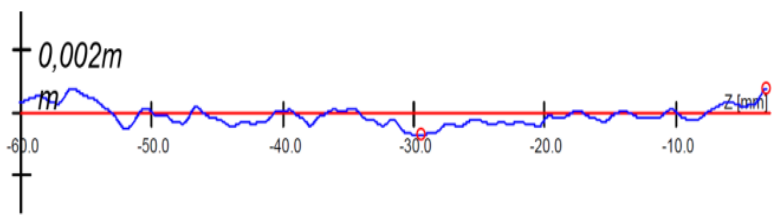

Fig. 3. Wall profile after the repair [4]
The inequality of mixing chamber wall was 0.045 $\mathrm{mm}$ before repair and $0.0016 \mathrm{~mm}$ after repair.

The pressure reducer that is in front of the nozzle can adjust pressure of primary flow. With this pressure controller, an approximate value can be set. The accurate value of the pressure of primary air is read by the measuring software and the pressure sensor before the nozzle. Checking these values is enabled by an analog manometer.

A mechanism that allows to adjust the backpressure is at the end of discharge pipe. The backpressure range is from $0 \mathrm{kPa}$ to $60 \mathrm{kPa}$ (relative) with a $5 \mathrm{kPa}$ step. Schema of the ejector track is in Figure 5.

There are restrictions causing pressure differentials in the suction and discharge pipes $\Delta p$. Mass flow rate is calculated from these measured differences.

The relationship used to calculate the mass flow from the measured differential pressure is:

$$
\dot{m}=\frac{C}{\sqrt{1-\beta^{4}}} \varepsilon_{1} \frac{\pi}{4} d_{c}^{2} \sqrt{2 \Delta p \varrho_{1}} .
$$

For the factor $\mathrm{C}$ following formula has been used:

$$
\begin{gathered}
C=0,5961+0,0261 \beta^{2}-0,216 \beta^{8}+0,000521\left(\frac{10^{6} \beta}{R e_{D}}\right)^{0,7}+ \\
{\left[0,0188+0,0063\left(\frac{19000 \beta}{R e_{D}}\right)^{0,8}\right] \beta^{3,5}\left(\frac{10^{6}}{R e_{D}}\right)^{0,3} .}
\end{gathered}
$$

All static pressures were measured using Kulite rapid response sensors, differential pressure on the apertures were measured using differential sensors.

From measurement we have acquired the characteristic of the ejector, which should ideally look similar to one in the Figure 4. It is a prerequisite for an infinitely small back-pressure step with sensors with infinite precision are used.

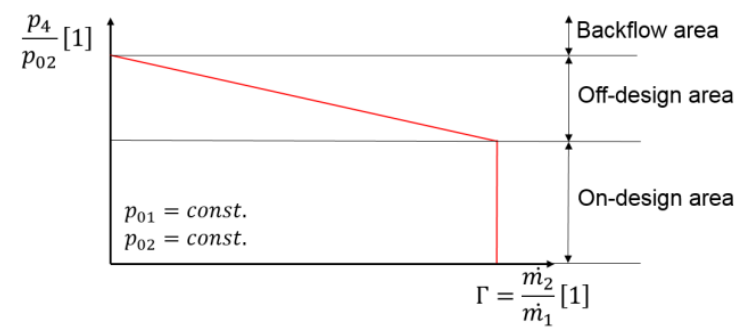

Fig. 4. Ideal shape of the ejector characteristic

These characteristics were obtained for each nozzle exit possition and for each of the stagnation pressure of the primary air. The possition of the nozzle exit significantly influences the intake quantity of the driven air and thus the operation of the whole ejector. 


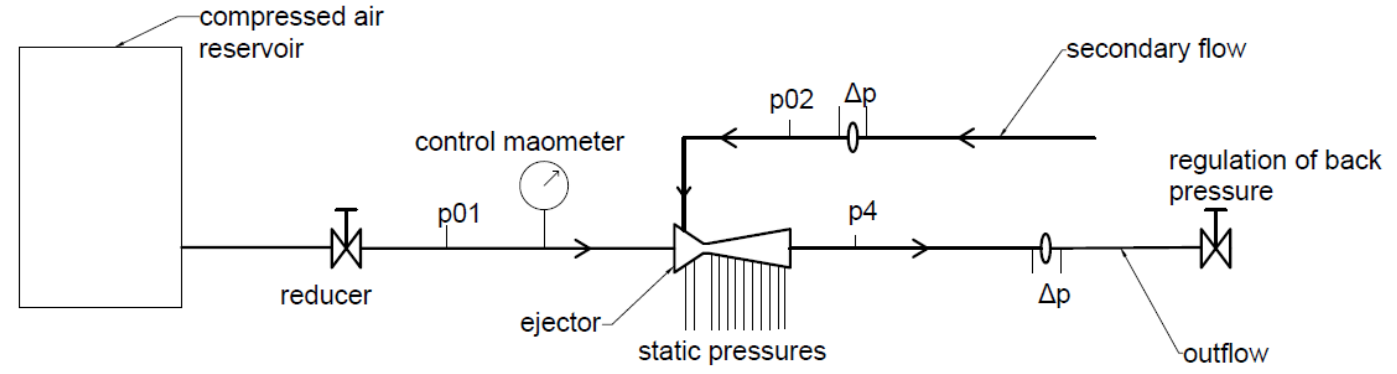

Fig. 5. Measurement schema of the ejector track

The entire ejector is composed from several parts. The mixing chamber is divided into short and long part, allowing measurements with different lengths of the mixing chamber. The individual parts are connected through locks to determine the exact position. All parts are clamped between two flanges.

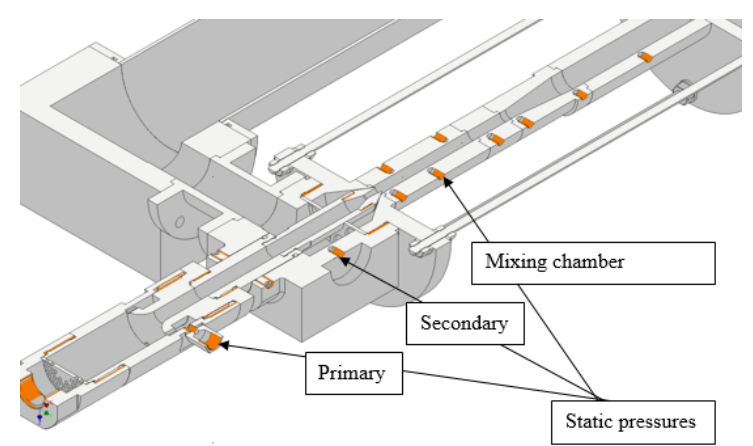

Fig. 6. The cut view of ejector model

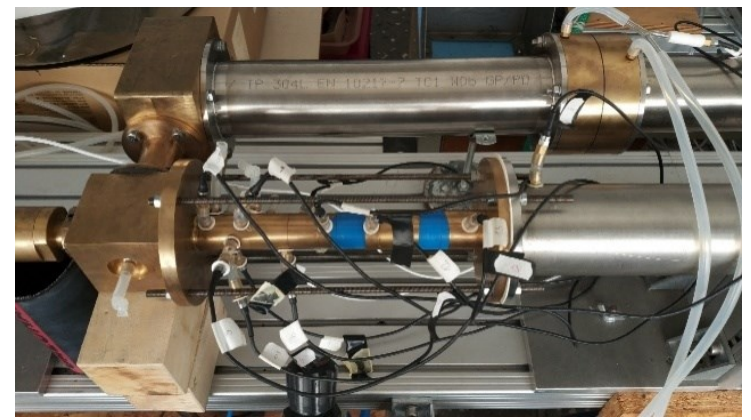

Fig. 7. Ejector assembly with pressure sensors

\section{Numerical simulation}

The experiment was also supported by numerical calculation. Since the measurements have been made for various primary nozzle positions nozzle position, also computational meshes were created for every primary nozzleposition.

The geometry of the model was created in SpaceClaim and the computational mesh was created in Ansys Meshing. All ejector models were designed as axisymmetric and each of them was appropriately partitioned so the computing mesh could be easyly created.The pattern of the partition of the model for nozzle position $2 \mathrm{~mm}$ is shown in Figure 8.

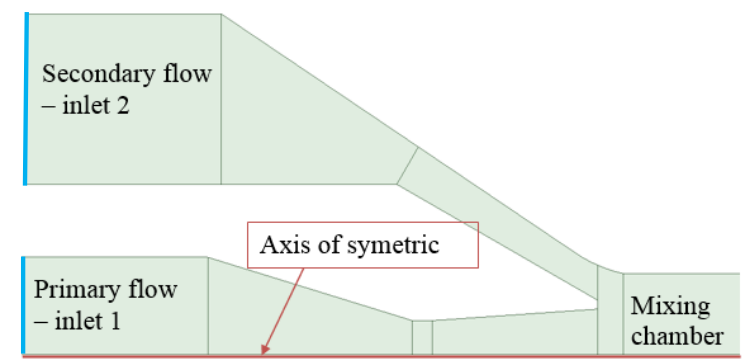

Fig. 8. Partitioning of the parts before creating the mesh, identification of the inputs and axis (for primary nozzle position $3 \mathrm{~mm}$ )

A 2D hexahedral mesh was created. The $y+$ value ranged in all calculations within the usability limits for a given turbulent model. The k-omega SST model was used as a turbulent model. When creating a mesh modeling, a boundary layer has been created, which is important for further settings of the calculation. The number of cells in these mesh ranged from 130000 to 150000 . The entire calculation was performed as transient in DensityBased mode for an axisymmetric model. To increase accuracy, the second order of upwind scheme was used in the calculation. Heat transfer through ejector walls was considered to be negligible. The turbulent intensity was set for all calculations to $2 \%$ and the hydraulic diameter was set to $0.01 \mathrm{~m}$.

The main dimensions of the ejector are shown in Table 1, the boundary conditions are shown in Table 2.

Table 1. Main geometry parameter of ejector

\begin{tabular}{|c|c|}
\hline Ejector parameter & dimension (mm) \\
\hline Nozzle throat diameter & 3.50 \\
\hline Nozzle exit diameter & 4.67 \\
\hline Mixing chamber diameter & 8.34 \\
\hline Mixing chamber length & 101.19 \\
\hline Diffuser outlet diameter & 16.00 \\
\hline Diffuser length & 38.10 \\
\hline
\end{tabular}


Table 2.Boundary conditions of the ejector model

\begin{tabular}{|c|c|}
\hline Boundary conditions & p (kPa) \\
\hline Primary inlet & 297, 397 \\
\hline Secondary inlet & 97 \\
\hline Outlet & $\begin{array}{ccc}97, & 107, & 117, \\
127, & 137, & 147 \\
& 157 & \end{array}$ \\
\hline
\end{tabular}

The governing equations can be written in the following forms:

$$
\begin{gathered}
p=\rho r T \\
\frac{\partial}{\partial t}\left(\rho u_{i}\right)+\frac{\partial}{\partial x_{j}}\left(\rho u_{i} u_{j}\right)=-\frac{\partial}{\partial x_{j}}\left(p \delta_{i j}\right)+\frac{\partial \tau_{i j}}{\partial x_{j}}, \\
\frac{\partial \rho}{\partial t}+\frac{\partial}{\partial x_{j}}\left(\rho u_{i}\right)=0
\end{gathered}
$$

$$
\frac{\partial}{\partial t}\left(\rho e_{0}\right)+\frac{\partial}{\partial x_{j}}\left(\rho u_{j} e_{0}+u_{j} p+q_{j}-u_{i} \tau_{i j}\right)=0 .
$$

As outlined above, these equations are solved by means of the commercial CFD software Ansys Fluent [3].

Geometry presented in previous article by by Kracik and Dvorak [2] is different, because mixing chamber was modified. We can notice the difference in the length of the mixing chamber, where the initial length was $98 \mathrm{~mm}$.

\section{Results and discussion}

Figure 9 and Figure 10 represent measured and calculated values. The lines through which the points are interposed represent the ideal ejector characteristics.

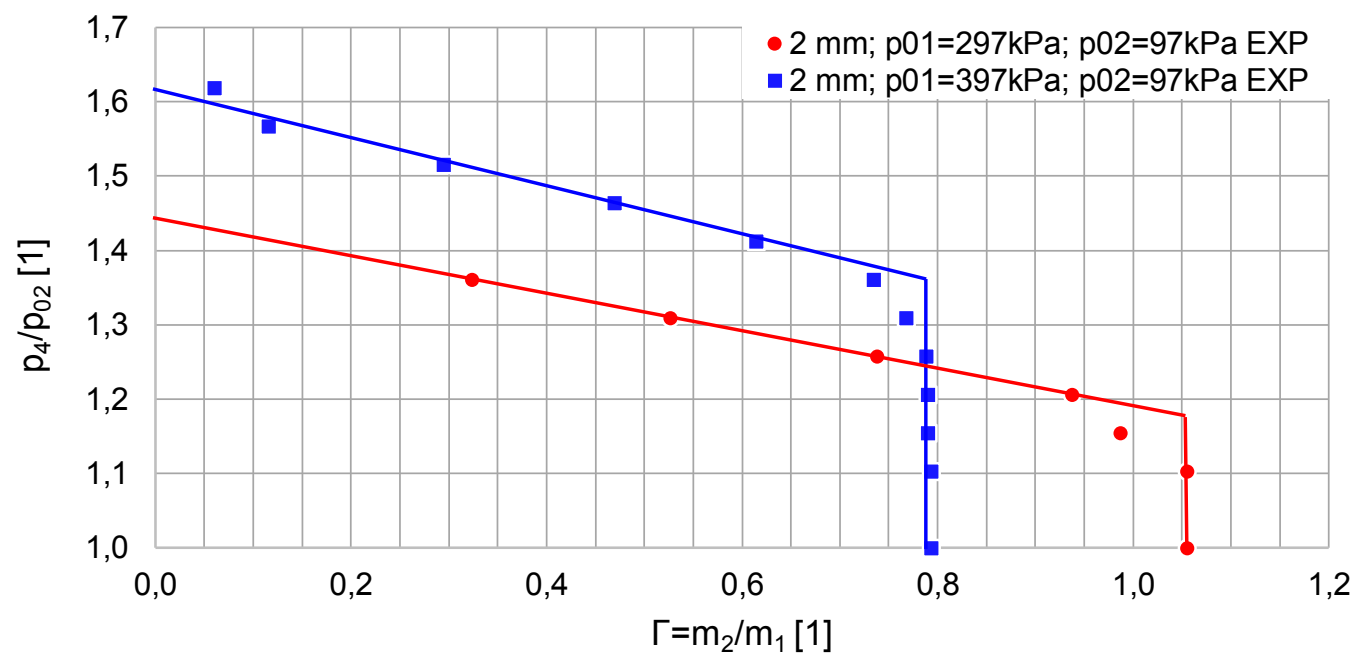

Fig. 9. Characteristics of the ejector

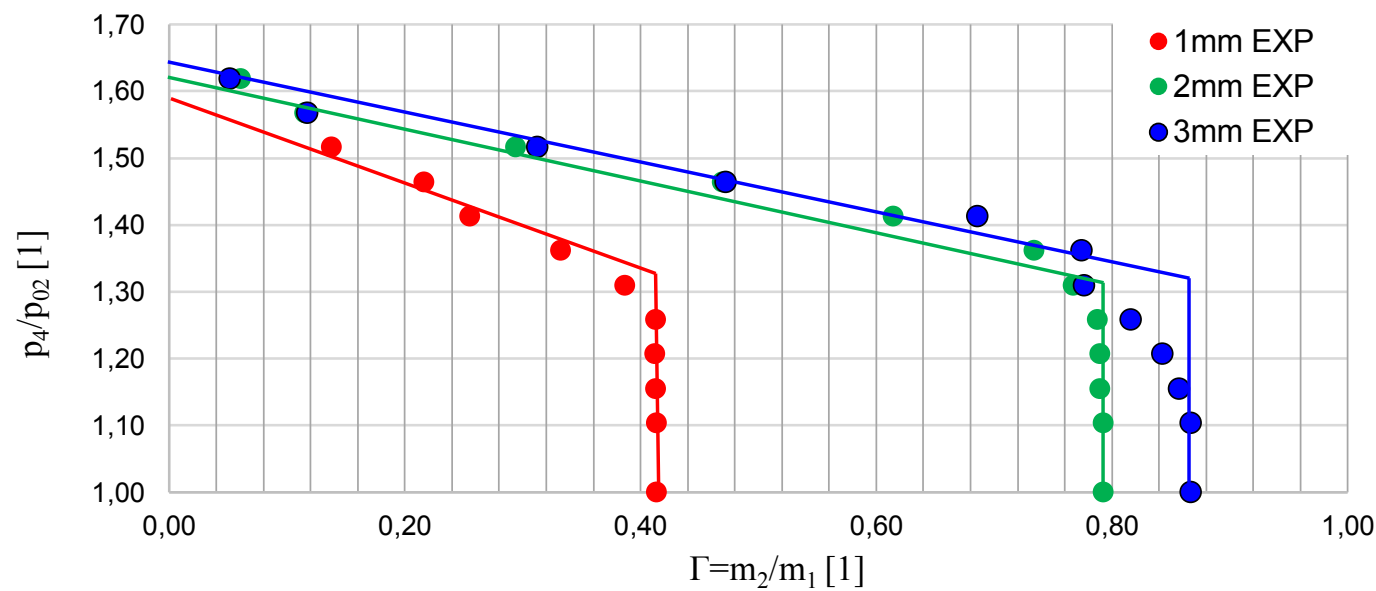

Fig. 10. Characteristics of the ejector - comparison of different primary nozzle position, $p_{01}=397 \mathrm{kPa}, p_{02}=97 \mathrm{kPa}$

The characteristic for one setting of the ejector with various values of primary pressure can be seen in Figure 9. As can be seen, the ejection coefficient increases when the amount of energy we deliver to the system (higher primary pressure) is increased. However, it is not always advisable to supply more 
energy to the system. By change in the position of the primary nozzle, the same ejection ratio can be obtain while maintaining the amount of energy supplied. This phenomenon can be seen by comparing Figures 9 and 10. The green characteristic in Figure 10 corresponds to the blue characteristic in Figure 9. When changing primary nozzle position from $2 \mathrm{~mm}$ to $3 \mathrm{~mm}$, the ejection performance changes. It should be kept in mind that the trend of increasing the ejection coefficient with NP is not constant.

Figure 11 compares the results obtained in the experiment and the CFD. The results correspond to the set-up of the boundary conditions listed above in Table 2. The results of CFD and experiment do not match much in the ejector on-design area. Results of the experiment for ejection ratio are overestimated to the results of the CFD method is interesting. With the assumption that CFD does not consider surface roughness, CFD results should show higher values than the experiment.

The following Figure 12 shows the static pressure distribution on the mixing chamber wall, the diffuser and the discharge pipe. In the area of the mixing chamber, the static pressures computed by the CFD method and experiment can be seen. This mismatch is due to the complicated mixing of primary flow and secondary flow. The static pressure difference between measured value and obtained from the CFD is lower than it was before modification of the mixing chamber. The reparation made it possible to get closer to the numerical solution.

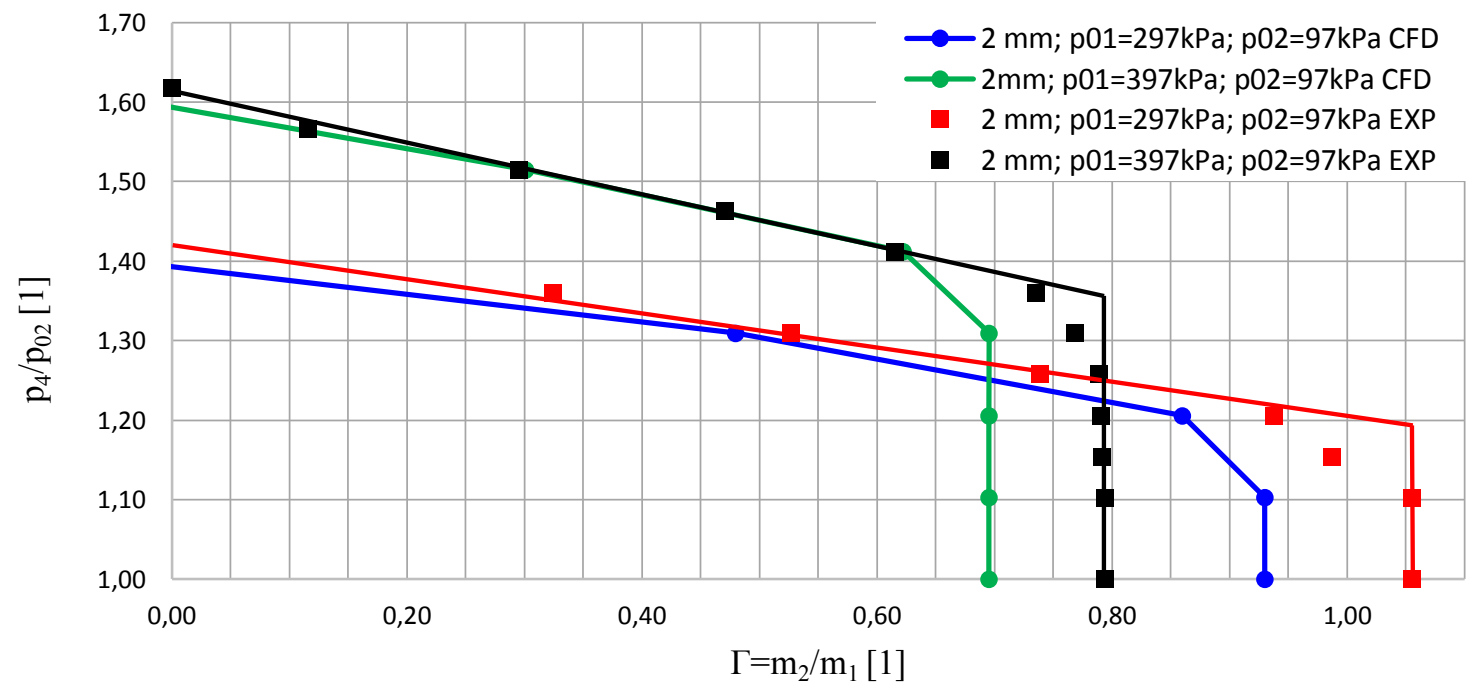

Fig. 11. Characteristic of ejector for NP $2 \mathrm{~mm}, \mathrm{CFD}$ and experiment for constant secondary pressure

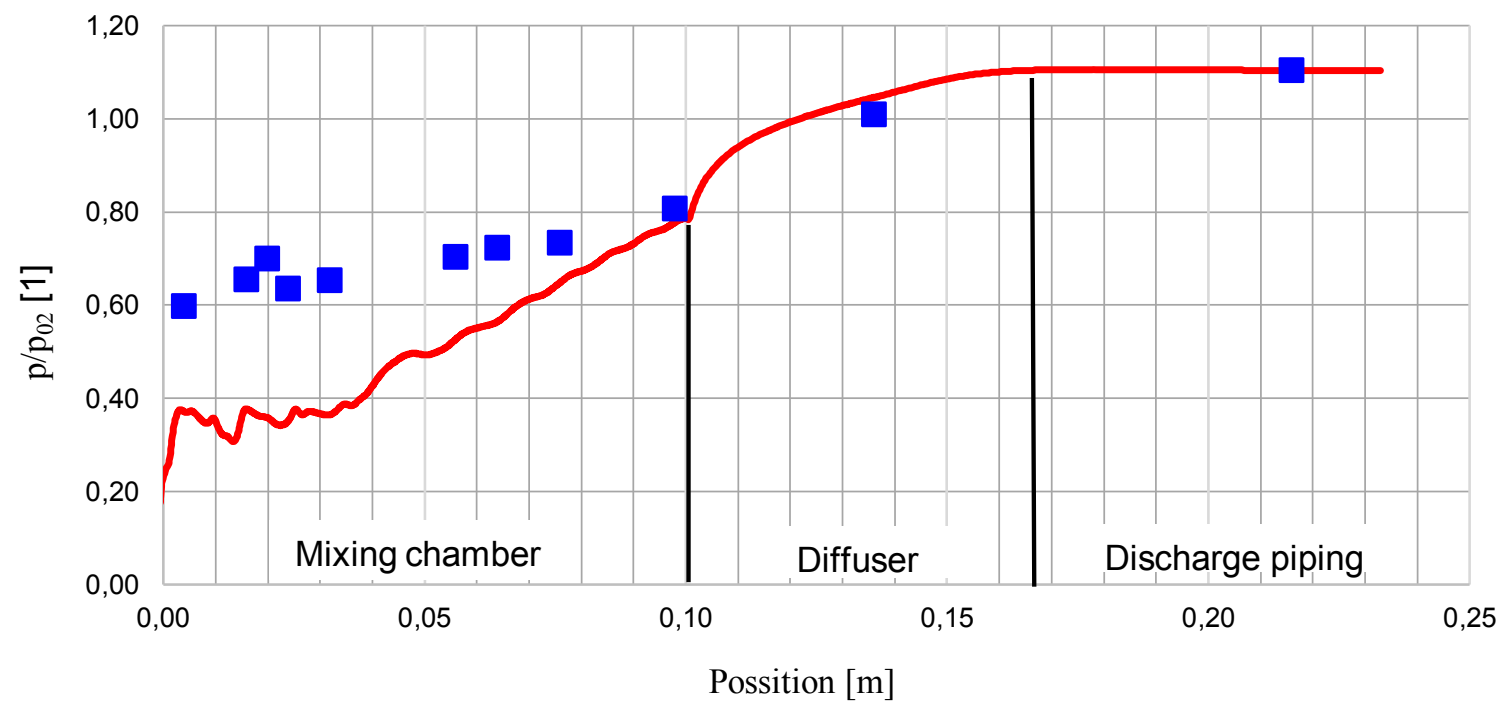

Fig. 12. Static pressure distribution, CFD (red line) and experiment (blue points) for NP $2 \mathrm{~mm}$ and $\frac{p_{4}}{p_{02}}=1.1$ 


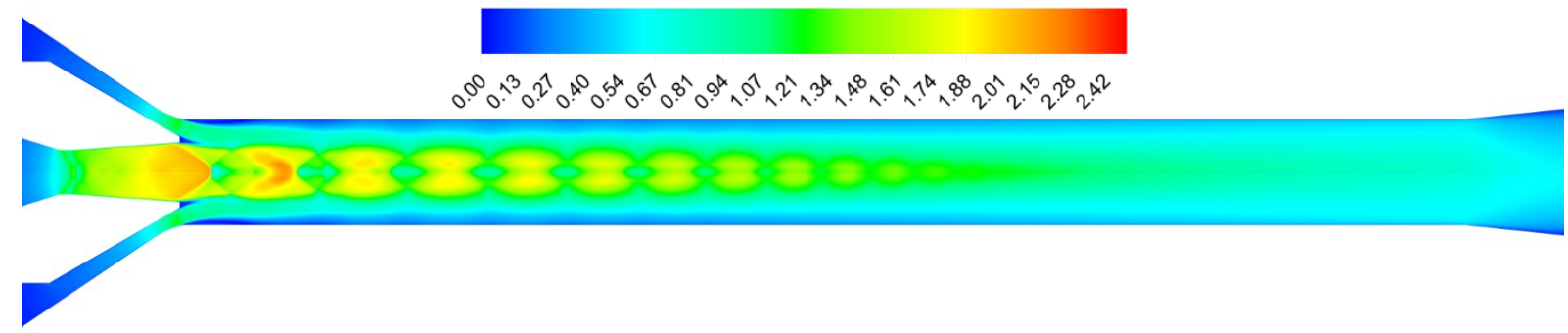

Fig. 13. Contours of Mach number- NP $2 \mathrm{~mm}, p_{01}=297 \mathrm{kPa}, p_{02}=97 \mathrm{kPa}, \frac{p_{4}}{p_{02}}=1.1$

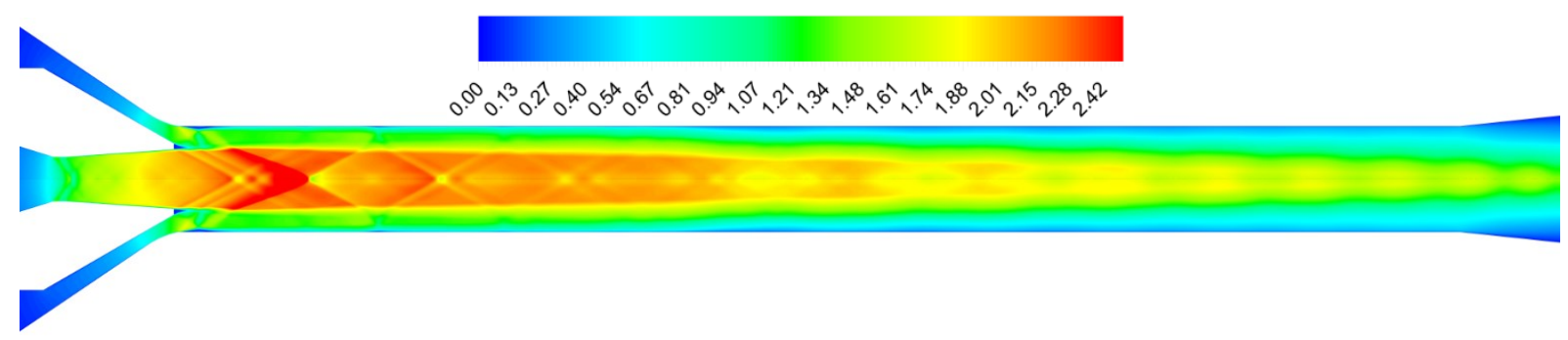

Fig. 14. Contours of Mach number $-\mathrm{NP} 2 \mathrm{~mm}, p_{01}=397 \mathrm{kPa}, p_{02}=97 \mathrm{kPa}, \frac{p_{4}}{p_{02}}=1.1$

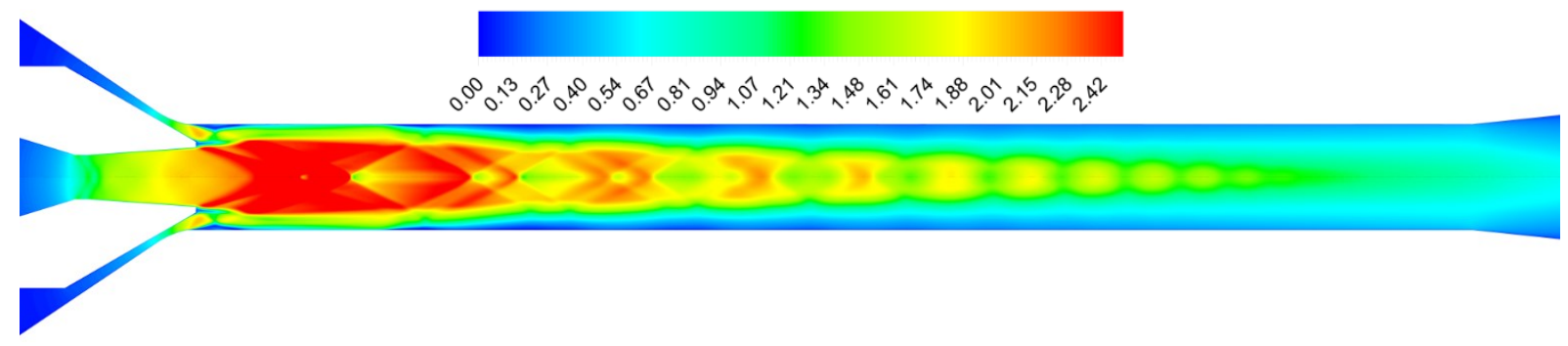

Fig. 15. Contours of Mach number $-\mathrm{NP} 1 \mathrm{~mm}, p_{01}=397 \mathrm{kPa}, p_{02}=97 \mathrm{kPa}, \frac{p_{4}}{p_{02}}=1.1$

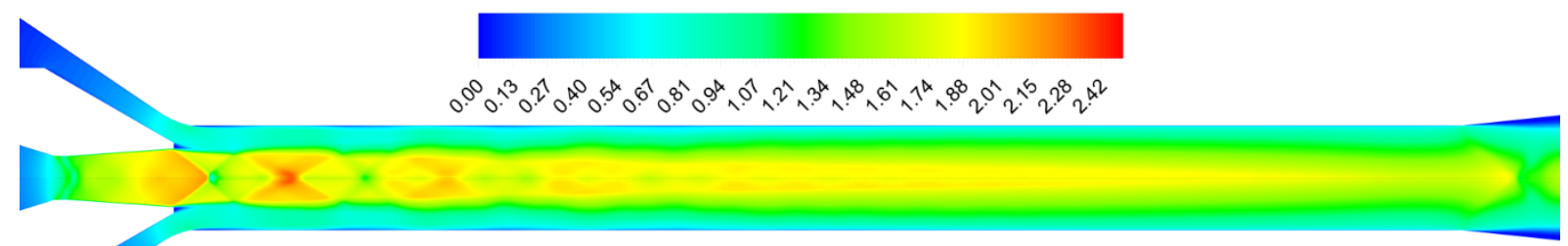

Fig. 16. Contours of Mach number $-\mathrm{NP} 3 \mathrm{~mm}, p_{01}=397 \mathrm{kPa}, p_{02}=97 \mathrm{kPa}, \frac{p_{4}}{p_{02}}=1.1$

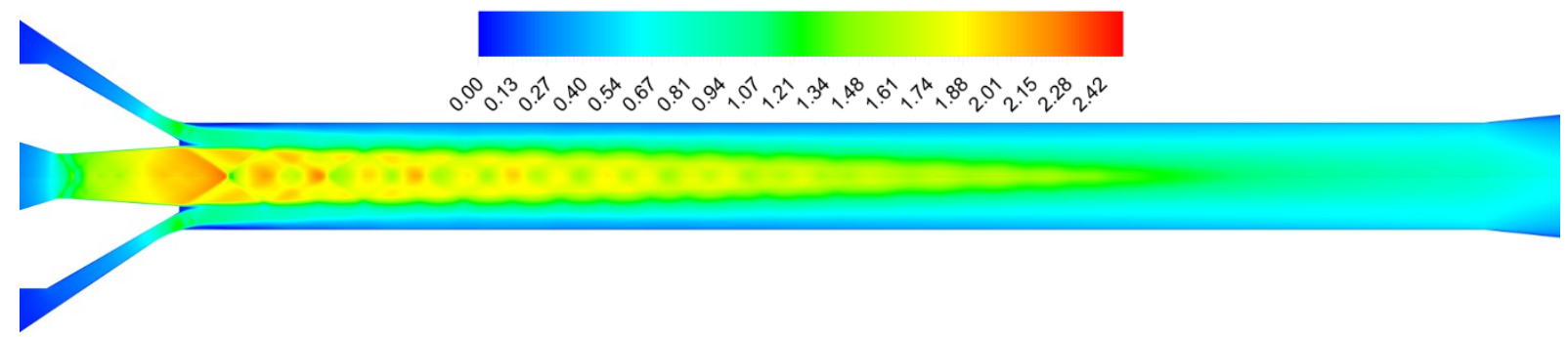

Fig. 17. Contours of Mach number $-\mathrm{NP} 2 \mathrm{~mm}, p_{01}=397 \mathrm{kPa}, p_{02}=97 \mathrm{kPa}, \frac{p_{4}}{p_{02}}=1.3$ 


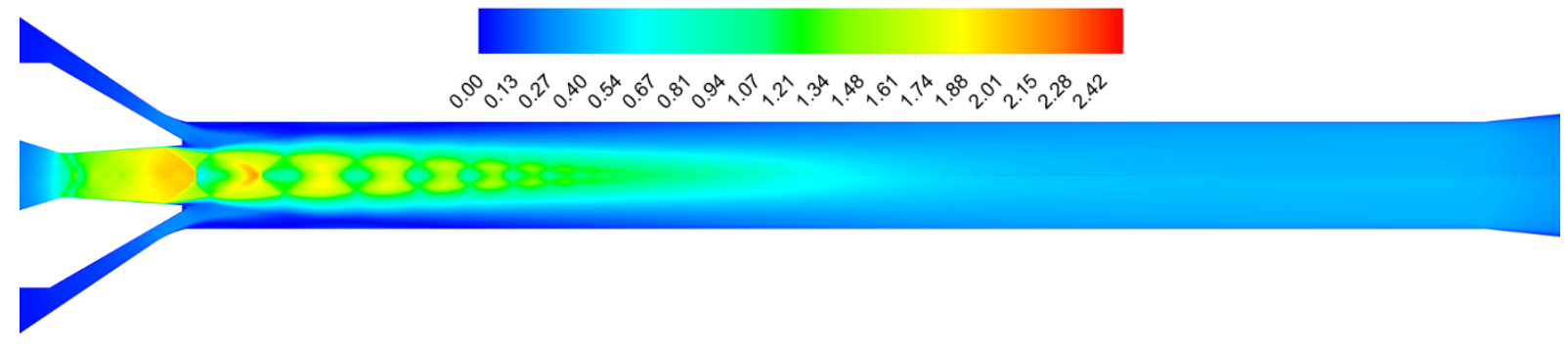

Fig. 18. Contours of Mach number $-\mathrm{NP} 2 \mathrm{~mm}, p_{01}=397 \mathrm{kPa}, p_{02}=97 \mathrm{kPa}, \frac{p_{4}}{p_{02}}=1.5$

In Figure 13 and Figure 14 we can compare the effect of primary pressure. The magnitude of this pressure influences both the primary and secondary mass flow rates. It also greatly affects possibility to overcome a higher back-pressure, as it is evident from characteristics in Figures 11 and 12, and mode of the nozzle.

Figures 14, 15 and 16 allow to compare the effect of primary nozzle position. It is also obvious that the value of the secondary stream significantly affects the nozzle mode and the operation of the entire ejector. For primary nozzle position set to $1 \mathrm{~mm}$, it can be noticed that the secondary stream enters the mixing chamber at supersonic speed and that the nozzle is in unexpanded mode. Detail is in Figure 19. With increasing nozzle position, the secondary mass flow that dampens the primary flow increases and changes the mode of the nozzle.

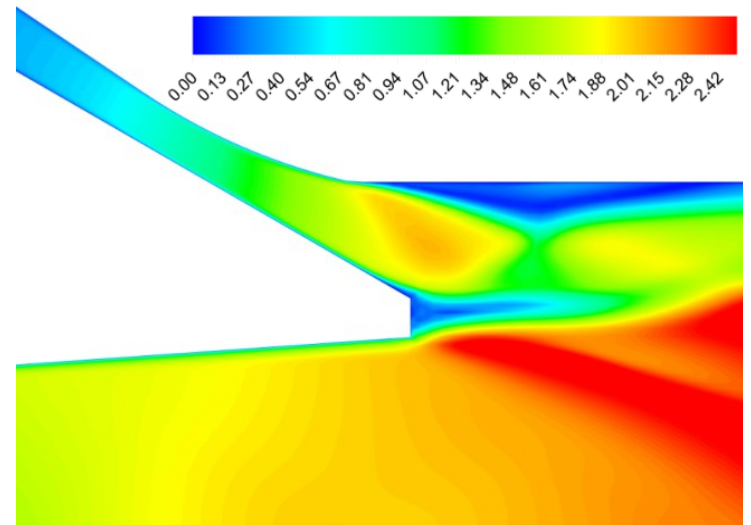

Fig. 19. Contours of Mach number - detail of supersonic secondary flow

The effect of the backpressure can be seen in Figures 14, 17 and 18. Figure 14 corresponds to the state in the characteristic when we are at the beginning of the ejector on-design. Figure 17 corresponds approximately to the state when the transition from the on-design area to the off-design area and Figure 18 corresponds to the backflow situation. The transition between the on-design area and the off-design area corresponds in the region around $\frac{p_{4}}{p_{02}}=1,35$ to set the primary pressure of $p_{01}=397 k P a$.

\section{Summary and conclusion}

This study dealt with experimentation on the ejector working with air with modified mixing chamber. As a support of the experiment, a total of 42 numerical simulations were performed for various primary pressure settings and primary nozzle position.

The highest ejection coefficient obtained in the experiment was set to $p_{01}=297 \mathrm{kPa}$ and NP $3 \mathrm{~mm}$, namely 1.14 . The highest critical backpressure value was to set the primary pressure to $397 \mathrm{kPa}$ for nozzle position of $1 \mathrm{~mm}$.

The problem that seems to be the most serious is the inconsistent static pressure values obtained from the experiment and the CFD. It would be advisable to address this issue and to fine-tune the measurement so that these differences are minimal and the experiment corresponds to the CFD.

This publication was written at the Technical University of Liberec as part of the project "Experimental and numerical investigation in applied fluid mechanics and energy devices, no. 21124" with the support of the Specific University Research Grant, as provided by the Ministry of Education, Youth and Sports of the Czech Republic in the year 2018.

\section{References}

1. Vaclav Dvorak, Flow in nozzles and diffusers, Technical University of Liberec, (2015). ISBN 978-80-7372-457-3

2. Jan Kracik and Vaclav Dvorak, EPJ WoC 143, (2017)

3. ANSYS. ANSYS FLUENT User's Guide. 15317, 2498 (2011)

4. TOS Varnsdorf, Measuring center

5. Michal Pavlas, Design and verification of supersonic ejectors, Master thesis (in Czech), Technical University of Liberec, (2017). 